\title{
Left atrial thrombus as an early consequence of blunt chest trauma
}

\author{
I R Mahy, A Al-Mohammad, R I Cargill
}

\begin{abstract}
Thromboembolism is rarely considered in discussions of the complications of blunt chest trauma. The few cases of thromboembolism that have been reported in this setting have occurred in association with significant myocardial damage. A previously fit 23 year old woman was admitted to the intensive care unit following a road traffic accident. A day later, left atrial thrombus was demonstrated by transoesophageal echocardiography in the absence of any other evidence of important myocardial injury. Anticoagulation with heparin was cautiously introduced in spite of her extensive injuries, and there were no consequent bleeding complications. At hospital discharge on day 18 she was entirely well. Full anticoagulation with warfarin was continued for a further eight weeks at which time follow up transoesophageal echocardiography showed complete resolution of the thrombus. (Heart 1998;79:198-199)
\end{abstract}

Keywords: blunt chest trauma; thromboembolism; left atrial thrombus; transoesophageal echocardiography

Reviews of the cardiac consequences of blunt chest trauma have typically focused on the haemodynamic effects of cardiac injury, or on the development of arrhythmias. Potential thromboembolic complications have received less attention. We report a case in which intracardiac thrombus occurred in the absence of other evidence of important myocardial contusion.

\section{Case report}

A previously fit 23 year old woman was admitted to the intensive care unit following a road traffic accident. She had been a passenger in the front seat (restrained by a seat belt) of a vehicle involved in head on collision. At the scene she was hypoxic and confused, and this persisted despite insertion of a chest drain for relief of a tension pneumothorax. She was intubated and ventilated before transfer.

At admission she was noted to have widespread bruising over the anterior chest wall, and there was extensive surgical emphysema. A haematoma over the occiput was also noted. Early computed tomography revealed multiple rib fractures, bilateral pneumothoraces and pneumomediastinum, extensive pulmonary consolidation and contusion, and an air-fluid level in the left sphenoidal sinus raising the possibility of a basal skull fracture (although no such fracture could be clearly identified). There was no evidence of major vessel disruption or intracerebral trauma.

The following day her condition had deteriorated with evidence of respiratory distress syndrome but stable haemodynamics without inotropic support. A loud pericardial rub was audible and the surface electrocardiogram showed changes typical of pericarditis. Transoesophageal echocardiography (Vingmed CFM 700, Horten, Norway) was performed to determine whether there was evidence of myocardial contusion, and to exclude aortic disruption.

The examination, 24 hours after the initial injury, revealed preserved left and right ventricular contraction, normal intracardiac valves, and a normal aorta. However, within the left atrium a highly mobile mass $(2 \times 1 \mathrm{~cm})$ was observed, apparently attached to the reflection of the left atrial appendage (fig 1). This was interpreted as being due to thrombus, with the possibility of an incidental cardiac tumour being considered less likely. Anticoagulation with heparin was cautiously introduced in spite of her extensive injuries, and there were no consequent bleeding complications. Serum enzyme analysis showed a peak creatine kinase $\mathrm{MB}$ of $17.2 \mathrm{U} / 1$ (normal < 9.5), which was $1.1 \%$ of the total creatine kinase.

Subsequent recovery allowed extubation on day 6 when the patient was noted to be ataxic and intermittently confused. Magnetic resonance imaging at this time showed no evidence of embolic complications but there was a small contusion in the right prefrontal lobe and some increased signal in the corpus callosum consistent with axonal injury.

At hospital discharge on day 18 she was entirely well. Full anticoagulation with warfarin was continued for a further eight weeks at which time follow up transoesophageal echocardiography showed complete resolution of the thrombus (fig 2).

\section{Discussion}

Intracardiac thrombus appears to be rare following blunt chest trauma. Three prospective studies predominantly using transthoracic
Accepted for publication 6 November 1997 


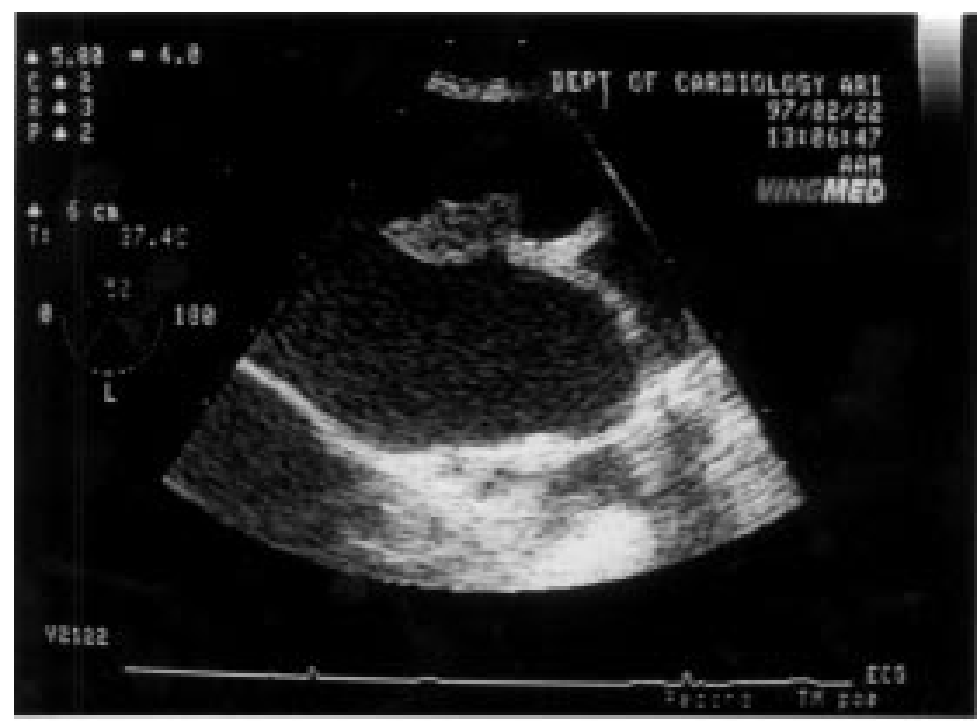

Figure 1 Transoesophageal echocardiogram of the left atrium 24 hours after the initial injury, demonstrating a tissue density mass that was highly mobile on real time imaging.

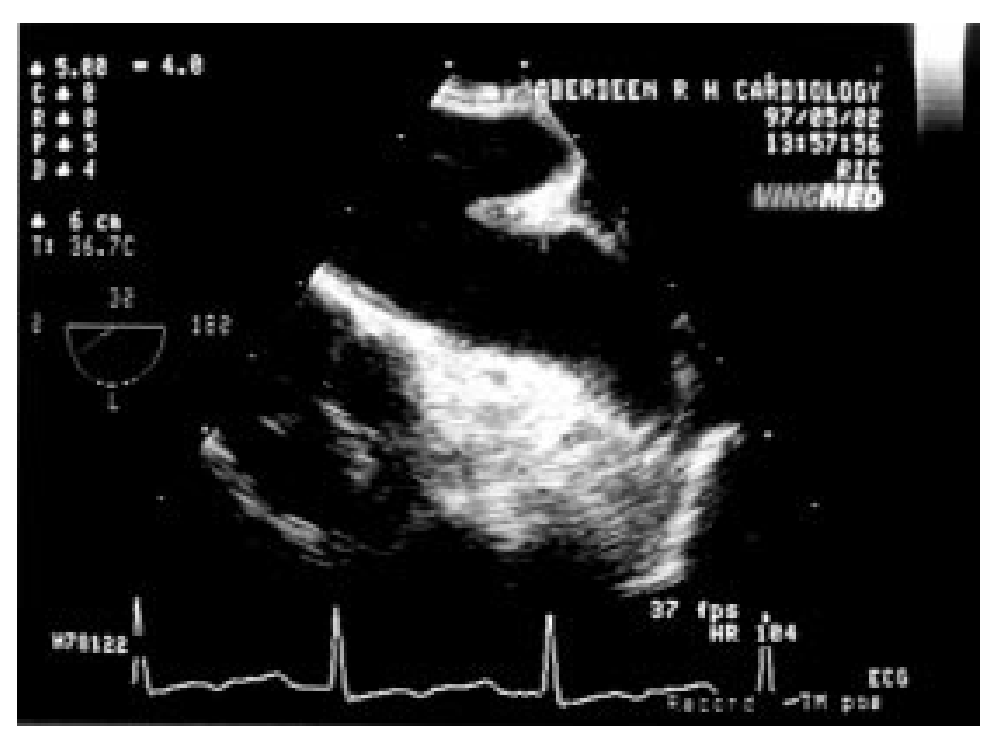

Figure 2 Transoesophageal echocardiogram of the left atrium after eight weeks of anticoagulation. The thrombus is no longer present.

echocardiography found no evidence of thrombus in 279 patients, ${ }^{1-3}$ and Chirillo et al found no cases of thrombus in their series of 134 consecutive transoesophageal studies for this indication, despite a large number of patients having evidence of myocardial contusion. ${ }^{4}$ Whether this is a consequence of the timing of these studies early after the initial injury is unclear, but a recent authoritative review of blunt cardiac trauma does not mention the potential for intracardiac thrombus formation in its discussion of this injury. ${ }^{5}$

The few reported cases of thromboembolism in this setting have occurred in association with evidence of substantial ventricular wall motion abnormality due to contusion. ${ }^{6}$ Grech et al described a case of pulmonary embolism attributed to mural thrombus within a contused right ventricle, although the thrombus was not imaged within the heart and alternative sources of embolism could not be ruled out. ${ }^{7}$ Rechavia et al describe a case of protruding left ventricular thrombus related to an area of wall motion abnormality several days after the initial injury, and cite two further similar cases of ventricular thrombus. ${ }^{8}$ Nishioka et al demonstrated thrombus in the right ventricular outflow tract following a gunshot wound that penetrated the epicardial surface of the heart but did not disrupt the intima. ${ }^{9}$ To our knowledge, however, left atrial thrombus has not been previously reported in association with blunt chest trauma, and in our case no evidence of major ventricular myocardial damage could be demonstrated.

The potential risk for embolisation is likely to differ when the thrombus lies mobile within the atrium rather than murally in association with a myocardial contusion. In the context of multiple injury such thrombus is a therapeutic problem, with the risks of bleeding offset against those of embolisation. Our therapeutic approach using low level anticoagulation may not be appropriate in all such cases. Alternatives are either open removal of the thrombus or expectant management and reliance on natural thrombolysis.

This case is unusual in several respects. It is the first case of left atrial thrombus demonstrated by echocardiography after blunt chest trauma, and is exceptional in that there was no other echocardiographic evidence of cardiac injury in association with this finding. A favourable clinical outcome with a conservative approach to this high risk situation is documented. Clinicians should remain alert to the possibility of intracardiac thrombus formation even in the absence of other markers of cardiac injury.

We thank Mr R R Jeffrey for permission to report this case.

1 Fabian TC, Mangiante EC, Patterson CR, et al. Myocardial contusions in blunt trauma: clinical characteristics, means of diagnosis and implications for patient management. $\mathcal{J}$ Trauma 1988;28:50-7.

2 Hiatt JR, Yeatman LA, Child JS. The value of echocardiography in blunt chest trauma. F Trauma 1988;28:914-22.

3 Helling TS, Duke P, Beggs CW, et al. A prospective evaluation of 68 patients suffering blunt chest trauma. F Trauma 1989;29:961-6.

4 Chirillo F, Totis O, Caverzerani A, et al. Usefulness of transthoracic and transoesophageal echocardiography in recognition and management of cardiovascular injuries after blunt chest trauma. Heart 1996;75:301-6.

5 Pretre R, Chilcott M. Current concepts: blunt trauma to the heart and great vessels. N Engl f Med 1997;336:626-32.

6 Timberlake GA, McSwain NE. Thromboembolism as a consequence of myocardial contusion-a new capricious consequence of myocardial contusion.

7 Grech ED, Dodds PA, Perry RA. Delayed complications after myocardial contusion. Br Heart $\mathcal{f}$ 1992;68:529-30.

after myocardial contusion. Br Heart f 1992;68:529-30.
8 Rechavia E, Imbar S, Birnbaum Y, et al. Protruding left venRechavia E, Imbar S, Birnbaum Y, et al. Protruding left ven-
tricular thrombus formation following blunt chest trauma. Am Heart f 1993;125:893-6.

9 Nishioka T, Fontana G, Luo H, et al. Intracardiac thrombus formation associated with a non-penetrating gunshot wound of the right ventricular outflow tract demonstrated by transoesophageal echocardiography. Am Heart $\mathcal{F}$ 1996; 132:1265-7. 